\title{
Phosphorylation of human INO80 is involved in DNA damage tolerance.
}

Dai Kato ${ }^{1}$, Mayumi Waki ${ }^{1}$, Masaki Umezawa ${ }^{1}$, Yuka Aoki ${ }^{1}$, Takahiko Utsugi ${ }^{2}$, Masaya Ohtsu ${ }^{1}$, and Yasufumi Murakami $^{1,2}$

${ }^{1}$ Department of Biological Science and Technology, Faculty of Industrial Science and Technology, Tokyo University of Science, 2641 Yamazaki, Noda, Chiba 278-8510, Japan

${ }^{2}$ Bio Matrix Research Inc. 105 Higashifukai, Nagareyama, Chiba 275-0101, Japan

Correspondence to;

Dr. Yasufumi Murakami

Biological Science and Technology

Faculty of Industrial Science and Technology

Tokyo University of Science

2641 Yamazaki, Noda-shi, Chiba 278-8510, JAPAN

Tel: 81-4-7124-1501 ex4408

FAX 81-4-7122-1360

Email: yasufumi@rs.noda.tus.ac.jp 


\begin{abstract}
Double strand breaks (DSBs) are the most serious type of DNA damage. DSBs can be generated directly by exposure to ionizing radiation or indirectly by replication fork collapse. The DNA damage tolerance pathway, which is conserved from bacteria to humans, prevents this collapse by overcoming replication blockages. The INO80 chromatin remodeling complex plays an important role in the DNA damage response. The yeast INO80 complex participates in the DNA damage tolerance pathway. The mechanisms regulating yINO80 complex are not fully understood, but yeast INO80 complex are necessary for efficient proliferating cell nuclear antigen (PCNA) ubiquitination and for recruitment of Rad18 to replication forks. In contrast, the function of the mammalian INO80 complex in DNA damage tolerance is less clear. Here, we show that human INO80 was necessary for PCNA ubiquitination and recruitment of Rad18 to DNA damage sites. Moreover, the C-terminal region of human INO80 was phosphorylated, and overexpression of a phosphorylation-deficient mutant of human INO80 resulted in decreased ubiquitination of PCNA during DNA replication. These results suggest that the human INO80 complex, like the yeast complex, was involved in the DNA damage tolerance pathway and that phosphorylation of human INO80 was involved in the DNA damage tolerance pathway. These findings provide new insights into the DNA damage tolerance pathway in mammalian cells.
\end{abstract}

\title{
Keywords
}

chromatin remodeling; INO80; DNA damage tolerance; PCNA; ubiquitination; DNA repair 


\section{Introduction}

DNA in living cells is highly vulnerable to endogenous and environmental damage. If damage to DNA is not repaired accurately, the damage may cause mutations, which can promote aging or lead to cancerous cells. Double strand breaks (DSBs) are the most serious type of DNA damage. Defects in DSB repair lead to genomic instability and tumorigenesis.

DSBs can be generated directly by exposure to ionizing radiation or indirectly by replication fork collapse. Bulky adducts, such as UV-induced cyclobutane pyrimidine dimers, in template DNA present a barrier to inhibit DNA synthesis by blocking the major eukaryotic replicative polymerases, including DNA polymerase $\delta$. Therefore, during DNA replication, unrepaired DNA damage may inhibit the progression of replication forks, and this inhibition may be converted to more severe secondary damage, such as DSBs [1]. Recently, it has become clear that cells, from bacteria to human, possess very important postreplication repair (PRR) to overcome such replication blockages [2].

In Saccharomyces cerevisiae, RAD6 and RAD18 are two of the most important genes involved in PRR, and mutation of either gene results in hypersensitivity to UV light and loss of UV-induced mutagenesis [3]. Rad6 is an ubiquitin-conjugating enzyme (E2) [4]; Rad18 is an ubiquitin-ligating enzyme (E3), and the interaction between Rad6 and Rad18 is essential for PRR [5,6]. Proliferating cell nuclear antigen (PCNA), which is a DNA polymerase sliding clamp involved in DNA synthesis and repair, is ubiquitinated in a Rad6- and Rad18-dependent manner, and monoubiquitination of PCNA is necessary for translesion DNA synthesis by polymerase $\eta$ in yeast cells $[7,8]$.

The genes responsible for PRR are highly conserved throughout evolution. Human PCNA is also monoubiquitinated in a RAD18-dependent manner at stalled replication forks by UV-induced lesions $[9,10]$. These findings indicate that the basic mechanism of PRR is conserved from yeast to mammals.

The INO80 chromatin complex is involved in DNA repair. Genomic DNA is generally folded and packaged along with histones as chromatin in nuclei of eukaryotes, but highly condensed chromatin presents a barrier against access of DNA repair proteins to sites of DNA damage. Histone modifications and ATP-dependent chromatin remodeling are the major mechanisms that alter chromatin structure, and both are important for DNA damage responses and DNA repair [11]. The yeast INO80 (yINO80) chromatin remodeling complex is recruited to DSBs via direct interaction of the Nhp10 or Arp4 
subunits with phosphorylated H2A, and yINO80 participates in DNA end processing in homologous recombination (HR)

repair [12-14]. We previously reported that the mammalian INO80 complex is recruited to sites of DNA damage in an ARP8-dependent manner, and this mechanism of recruitment differs from that of the yeast INO80 complex [15].

The mechanisms regulating yINO80 complex are not understood fully, but yINO80 complex are necessary for efficient PCNA ubiquitination and for recruitment of Rad18 to replication forks [16]. On the other hands, whether mammalian INO80 complex function in DNA damage tolerance remains unknown.

Here, we demonstrate that hINO80, core component of hINO80 complex, is involved in ubiquitination of PCNA. Additionally, the C-terminal region of hINO80 was phosphorylated, and this phosphorylation was necessary for the DNA damage tolerance pathway.

\section{Materials and Methods}

\section{Cell culture}

HeLa cells and HEK293T cells were grown at $37^{\circ} \mathrm{C}$ in Dulbecco's Modified Eagle Medium (Invitrogen, Carlsbad, CA) supplemented with 10\% fetal bovine serum (Invitrogen), $2 \mathrm{mM} \mathrm{L-glutamine} \mathrm{(Invitrogen),} \mathrm{and} \mathrm{1 \%} \mathrm{penicillin} \mathrm{streptomycin}$ (Invitrogen) in a $5 \% \mathrm{CO}_{2}$ environment.

\section{Antibodies}

A monoclonal antibody against mammalian INO80 was obtained from Biomatrix Research Inc. (Chiba, Japan). Mouse anti-PCNA (sc-56) was purchased from Santa Cruz Biotechnology. Mouse anti-Rad18 (3H7) was purchased from Abnova (Taipei city, Taiwan). Mouse anti-FLAG M2 was purchased from Sigma Aldrich (St. Louis, MO). Mouse anti-GAPDH (ab8245) was purchased from Abcam (Cambridge, U.K.). HRP-conjugated goat anti-mouse IgG, and Alexa Fluor 488 goat anti-mouse $\mathrm{IgG}$ were purchased from Invitrogen. 
Plasmid construction, transfection, Western blot analysis, immunofluorescent staining, $\lambda$-phosphatase treatment, and Cell cycle synchronization

These methods are as described in the supplementary information.

Both sequences of primers and siRNA sequences used in this study were described as tables in the supplementary information.

\section{Results}

\section{Human INO80 is involved in PCNA ubiquitination.}

PCNA is ubiquitinated in a RAD6- and RAD18-dependent manner, and ubiquitination of PCNA is essential for PRR in many eukaryotes [7-10]. Previous studies showed that the yeast INO80 complex participates in the DNA damage tolerance pathway [16]. However, whether the mammalian INO80 complex also participates in this pathway is still unknown. To elucidate the cellular functions of hINO80, we treated HeLa cells with a non targeting control (NC) siRNA or with a specific hINO80 siRNA that resulted in approximately 70\% knockdown of hINO80 protein expression (Fig. 1A). We analyzed cell-cycle distribution of siRNA-treated cells and found no significant alteration in cell-cycle distribution of hINO80-knockdown cells relative to control cells (Supplementary Fig. 1).

To investigate whether hINO80 is necessary for activation of the primary step in DNA damage tolerance pathway, we examined PCNA ubiquitination following depletion of hINO80 using siRNA. The long exposure of Western blotting visualized ubiquitinated PCNA which was high molecular weight (Fig. 1B). Depletion of hINO80 significantly reduced PCNA ubiquitination (Fig. 1B). To exclude the possibility that the reduction of PCNA ubiquitination was an off-target effect of siRNA, we performed rescue experiments by expressing exogenous hINO80. Expression of exogenous hINO80 rescued PCNA ubiquitination following hINO80 depletion by siRNA (Fig. 1C). Taken together, these results indicate that hINO80 is important for efficient PCNA ubiquitination. 


\section{Human INO80 is necessary for recruitment of Rad18}

Previous studies indicate that PCNA is monoubiquitinated in a RAD18-dependent manner in both yeast and mammalian cells [7-10]. In S.cerevisiae, the yINO80 complex is required for efficient recruitment of Rad18, which initiates the PCNA ubiquitination pathway [16]. In mammalian cells, UV irradiation induces relocalization of RAD18 in the nucleus, such that RAD18 co-localizes with PCNA [10]. To investigate expression and localization of Rad18 following depletion of hINO80, we treated HeLa cells with hINO80 siRNA or with NC siRNA. Western blot analysis of these cells revealed that there was not a marked difference in expression of RAD18 protein (Fig. 2A). Immunostaining using anti-RAD18 antibody after UV irradiation revealed that siRNA-mediated depletion of hINO80 expression significantly reduced nuclear dots intensity of RAD18 (Fig. 2B, C). These results suggest that hINO80 is required for efficient recruitment of RAD18 that initiates PCNA ubiquitination at sites of DNA damage.

\section{The $\mathrm{C}$ terminal region of hINO80 is phosphorylated.}

Previous studies indicate that multiple protein modifications, such as phosphorylation, ubiquitylation, and acetylation, play an important role in signal transduction that activate the DNA damage response which includes cell cycle arrest, cell cycle checkpoint activation, DNA repair, or apoptosis [17]. In S.cerevisiae, the Ies4 subunit of the yINO80 complex is phosphorylated by Mec1 and Tel1, and the yINO80 complex is involved in checkpoint response [18]. Recently, a comparison between mammalian and yeast INO80 complexes revealed that these complexes possess both conserved subunits and several non-conserved subunits [19]. Interestingly, the Ies4 subunit of yINO80, which functions in checkpoint activation, is not present in the mammalian INO80 complex [18]. Based on several mass spectrometry studies, hINO80 core subunit is subject to post-translational modification [20,21]; therefore, we analyzed post-translational modification of hINO80. Western blotting with the anti-INO80 antibody showed a faint minor band which was migrating faster than main band in Figure 3A. As this band was not always clearly visible in western blot analyses, the appearance of the minor band might be due to a non-specific interaction of the antibody. To determine whether endogenous hINO80 was phosphorylated, 
we treated total cell extract with $\lambda$-phosphatase. Phosphatase treatment altered migration of hINO80 toward decreasing its molecular weight (Fig. 3A). This result suggests that endogenous hINO80 is phosphorylated.

To confine a region of hINO80 was subject to phosphorylation, we transfected $293 \mathrm{~T}$ cells with constructs that each encoded a different FLAG-tagged hINO80 deletion mutant (Fig. 3B). Western blot analyses revealed that there were two forms of the hINO80 C-terminal segment (Fig. 3C). To verify that these two different forms of the hINO80 C-terminal segment resulted from differences in phosphorylation, we transfected 293T cells with the construct that encoded the FLAG-tagged C-terminus of hINO80 and treated total cell extract from these cells with $\lambda$-phosphatase. Following phosphatase treatment, only one form of the hINO80 C-terminus was detected. This form of the hINO80 C-terminus seemed to correspond to the faster migrating form, suggesting that hINO80 C-terminus is phosphorylated (Fig. 3D).

Based on several mass spectrometry studies, the candidate phosphorylated sites in hINO80 are Ser 1490, Ser 1512, Ser 1516, and Thr 1550. These residues are located in the C terminal domain of hINO80 [20,21]. None of these phosphorylation sites have been functionally characterized. We made four phosphorylation-defective mutant FLAG-tagged C-terminal hINO80 constructs (PM1, PM2, PM3, PM4). In each mutant, Thr and/or Ser residues at phosphorylation sites were replaced with Ala residues (Fig. 3E). To analyze phosphorylation status of these hINO80 mutants, we transfected 293T cells with individual mutant constructs. Western blot analysis shows that there were two bands which are migrating close together in the each lane of PM3 and PM4 (Fig. 3F). These two bands were also visible in the lane of PM1. Although we currently do not have any further information on the nature of these two bands, we would like to continue characterization using a 2D-electrophoresis analysis. Cells expressing PM1 or PM2 had a slower migrating form of the protein. These slower migrating proteins were not observed in extracts from cells expressing PM3 or PM4 (Fig. 3F). These finding suggest that the hINO80C-terminus was subject to phosphorylation at either Ser 1512 or Ser 1516.

\section{The phosphorylation of hINO80 is involved in DNA damage tolerance.}

Recent study suggests that RAD18 is phosphorylated by CDC7, and this phosphorylation promotes recruitment of DNA polymerase $\eta$ to stalled replication forks [22]. Therefore, it is believed that the regulation of phosphorylation is important 
not only for the DSB repair pathway, but also for PPR pathway. To investigate whether phosphorylation of the hINO80 C-terminus is necessary for efficient ubiquitination of PCNA, we created a construct that encodes hINO80 in which Ala was substituted for all 4 residues (PM-hINO80). To compare expression of hINO80 and PM-hINO80, we transfected 293T cells with the FLAG-tagged hINO80 or FLAG-tagged PM-hINO80 construct. Western blot analysis revealed that there was not a marked difference in expression of hINO80 and PM-hINO80 (Supplementary Fig. 2A).

To determine whether phosphorylation of the hINO80 C-terminus was necessary for ubiquitination of PCNA during DNA replication, we synchronized the cell cycle by hydroxyurea (HU). We synchronized the cell cycle of HEK293T cells transfected with FLAG-tagged hINO80 or FLAG-tagged PM-hINO80; these cells were released from the induced G1/S-phase arrest using drug-free medium. These cells were harvested once every hour and processed for flow cytometry or for Western blotting. FACS analysis indicated that there was not a marked difference between cells that overexpressed hINO80 and those that overexpressed PM-hINO80 while they underwent DNA replication following removal of HU (Supplementary Fig. 2B). We next investigated PCNA ubiquitination in cells that overexpressed hINO80 or PM-hINO80. PCNA ubiquitination was lower in cells that overexpressed PM-hINO80 than in those that overexpressed hINO80 (Fig. 4A).This result suggests that phosphorylation of the hINO80 C-terminus was necessary for efficient PCNA ubiquitination during DNA replication.

PCNA ubiquitination is essential for PRR, and defects in the PRR cause an increase in the amount of DSB during DNA replication [1]; therefore, we next investigated the accumulation of the phosphorylated form of H2AX (a marker of DSB formation, $\gamma \mathrm{H} 2 \mathrm{AX}$ ) during DNA replication. In PM-hINO80-overexpressing cells, $\gamma \mathrm{H} 2 \mathrm{AX}$ accumulation was enhanced after release from HU (Fig. 4B). This finding indicated that hINO80 was involved in the DNA damage tolerance pathway and that this function of hINO80 was regulated by phosphorylation. 


\section{Discussion}

\section{Human INO80 is involved in the DNA damage tolerance pathway.}

Previous findings indicate that human INO80 is indirectly and directly involved in DNA repair. Mammalian INO80 facilitates HR-mediated DSB repair via expression of RAD51 and XRCC3 genes [23]. We previously reported that the mammalian INO80 complex is recruited to DNA damage sites in ARP8 dependent manner. This is an example of direct involvement of INO80 complex in DNA repair.

Human INO80 seems to be directly involved in PCNA ubiquitination. Here, we demonstrated that hINO80 is involved in ubiquitination of PCNA (Fig. 1B, C). However, expression of RAD18 protein, the enzyme that catalyzes ligation of ubiquitin to PCNA, was minimally affected by hINO80 depletion (Fig. 2A). Depletion of hINO80 expression significantly reduced nuclear dots intensity of RAD18 (Fig. 2B, C). This finding supports the hypothesis that hINO80 is directly related to PCNA ubiquitination. PCNA is ubiquitinated in a RAD6- and RAD18-dependent manner, and monoubiquitination of PCNA is essential for translesion synthesis by DNA polymerase $\eta$ in both human and yeast cells [7-10]. Our study shows that hINO80 was necessary for PCNA ubiquitination during DNA replication. Thus, our findings indicated that the INO80 complex was involved in the DNA damage tolerance pathway in human cells as well as in yeast cells.

Yeast INO80 is necessary for efficient recruitment of Rad18 to replication forks. Although it is unknown whether chromatin remodeling activity of the yINO80 complex is necessary for recruitment of Rad18, chromatin remodeling activity is necessary for PCNA ubiquitination [16]. Here, expression of RAD18 protein was not clearly affected by hINO80 depletion, but depletion of hINO80 significantly reduced RAD18 nuclear dots (Fig. 2). We could not detect an interaction between hINO80 and RAD18 (data not shown). Interestingly, a previous study revealed that hINO80 co-localizes with PCNA during the S phase, but not in an ATPase mutant of hINO80 [24]. These findings raise the possibility that access of RAD18 to DNA damage sites may require alteration of chromatin structure via the chromatin remodeling activity of hINO80.

Human INO80 promotes the removal of UV lesions via the nucleotide excision repair (NER) pathway[25]. During G1 phase, NER is the primary pathway that removes damage introduced into cellular DNA by UV and by a variety of chemical carcinogens. However, some lesions such as UV-induced cyclobutane pyrimidine dimers (CPDs) are repaired slowly by 
NER; consequently, unrepaired lesions can persist in DNA during DNA replication. Replicative DNA polymerases have very high fidelity and a stringent requirement for undamaged bases in their active sites. Therefore, almost all DNA lesions block progress of the replication fork. The DNA damage tolerance pathway plays an important role in overcoming such replication blockages, and this pathway is conserved from bacteria to humans. Our results could indicate that hINO80 is involved in the DNA damage tolerance pathway. This study and other studies suggest that hINO80 was a multifunctional protein that contributed to genome stability via multiple functions and during several cell cycle phases. In fact, previous studies indicate that the persistent depletion of hINO80 results in aneuploidy and structural chromosome abnormalities [24].

\section{Phosphorylation of human INO80 chromatin remodeling complex}

Here, we successfully characterized the role of hINO80 phosphorylation for the first time. The Ies4 subunit of the $S$. cerevisiae INO80 complex is phosphorylated by Mec1 and Tel1, and the yINO80 complex is involved in checkpoint responses [18]. The Ies4 subunit, which participates in checkpoint activation in yeast, is not present in the mammalian INO80 complex [19]. In humans, phosphorylation of hINO80 protein, a core subunit of the complex, may correspond to phosphorylation of the IES4 subunit in the yINO80 complex. Our study indicated that phosphorylation of a chromatin remodeling complex was necessary for the DNA damage tolerance pathway. These results revealed the importance of post-translational modification of a chromatin remodeling complex in this process. Phosphorylation-dependent alterations are occurring in the activation of the hSWI/SNF chromatin remodeling complex [26,27]. This study and other studies suggest that the chromatin-modifying complexes themselves, as well as histones, are also regulated by post-translational modification.

\section{Phosphorylation-dependent regulation of hINO80}

We show that phosphorylation of hINO80 is necessary for PCNA ubiquitination (Fig. 4A). However, the kinase that phosphorylates hINO80 was not identified. The regulation via phosphorylation is important in the DNA damage tolerance pathway. RAD18 is phosphorylated by $\mathrm{CDC} 7$, and this phosphorylation promotes efficient recruitment of polymerase $\eta$ to 
stalled replication forks [22]. S/T-P and S/T-Q are phosphorylation motifs for the Cdc7 kinase [28]. Our results also suggests that Ser1512 and Ser1516 of hINO80 are phosphorylated (Fig. 3), and that these residues are the S/T-P motif for Cdc7 phosphorylation. These findings support the hypothesis that CDC7 is the kinase that phosphorylates hINO80 in the DNA damage tolerance pathway.

Despite the established clinical importance of the DNA damage tolerance pathway in preventing human carcinogenesis [29, 30], details of this pathway are not well characterized. Here, we demonstrate that hINO80 is involved in the DNA damage tolerance pathway and that phosphorylation plays an important role in the pathway. These findings may contribute to the prevention of human carcinogenesis in the future.

\section{Acknowledgement}

This work has been supported by the grants supplied by the Ministry of Education, Culture, Sports, Science and Technology, the Japan Science and Technology Agency, and Bio Matrix Research Inc.

\section{Reference}

[1] J.E. Haber, DNA recombination: the replication connection, Trends Biochem. Sci. 24 (1999) 271-275.

[2] A.R. Lehmann, Replication of damaged DNA in mammalian cells: new solutions to an old problem, Mutat. Res. 509 (2002) 23-34.

[3] S. Broomfield, T. Hryciw, W. Xiao, DNA postreplication repair and mutagenesis in Saccharomyces cerevisiae, Mutat. Res. 486 (2001) 167-184.

[4] P. Sung, S. Prakash, L. Prakash, Mutation of cysteine-88 in the Saccharomyces cerevisiae RAD6 protein abolishes its ubiquitin-conjugating activity and its various biological functions, Proc. Natl. Acad. Sci. U.S.A. 87 (1990) 2695-2699.

[5] P. Sung, E. Berleth, C. Pickart, S. Prakash, L. Prakash, Yeast RAD6 encoded ubiquitin conjugating enzyme mediates protein degradation dependent on the N-end-recognizing E3 enzyme, EMBO J. 8(1991) 2187-2193. 
[6] V. Bailly, J. Lamb, P. Sung, S. Prakash, L. Prakash, Specific complex formation between yeast RAD6 and RAD18 proteins: a potential mechanism for targeting RAD6 ubiquitin-conjugating activity to DNA damage sites, Genes Dev. 8 (1994) 811-820.

[7] C. Hoege, B. Pfander, G.L. Moldovan, G. Pyrowolakis, S. Jentsch, RAD6-dependent DNA repair is linked to modification of PCNA by ubiquitin and SUMO, Nature 419 (2002) 135-141.

[8] P. Stelter, H.D. Ulrich, Control of spontaneous and damage-induced mutagenesis by SUMO and ubiquitin conjugation, Nature 425 (2003) 188-191.

[9] P.L. Kannouche, J. Wing, A.R. Lehmann, Interaction of human DNA polymerase eta with monoubiquitinated PCNA: a possible mechanism for the polymerase switch in response to DNA damage, Mol. Cell 14 (2004) 491-500.

[10] K. Watanabe, S. Tateishi, M. Kawasuji, T. Tsurimoto, H. Inoue, M. Yamaizumi, Rad18 guides poleta to replication stalling sites through physical interaction and PCNA monoubiquitination, EMBO J. 23 (2004) 3886-3896.

[11] M. Downey, D. Durocher, Chromatin and DNA repair: the benefits of relaxation, Nat. Cell Biol. 8 (2006) 9-10.

[12] J.A. Downs, S. Allard, O. Jobin-Robitaille, A. Javaheri, A. Auger, N. Bouchard, S.J. Kron, S.P. Jackson, J. Cote, Binding of chromatin-modifying activities to phosphorylated histone H2A at DNA damage sites, Mol. Cell 16 (2004) 979-990.

[13] A.J. Morrison, J. Highland, N.J. Krogan, A. Arbel-Eden, J.F. Greenblatt, J.E. Haber, X. Shen, INO80 and gamma-H2AX interaction links ATP-dependent chromatin remodeling to DNA damage repair, Cell 119 (2004) $767-775$.

[14] H. van Attikum, O. Fritsch, B. Hohn, S.M. Gasser, Recruitment of the INO80 complex by H2A phosphorylation links ATP-dependent chromatin remodeling with DNA double-strand break repair, Cell 119 (2004) 777-788.

[15] S. Kashiwaba, K. Kitahashi, T. Watanabe, F. Onoda, M. Ohtsu, Y. Murakami, The mammalian INO80 complex is recruited to DNA damage sites in an ARP8 dependent manner, Biochem. Biophys. Res. Commun. 402 (2010) 619-625.

[16] K.B. Falbo, C. Alabert, Y. Katou, S. Wu, J. Han, T. Wehr, J. Xiao, X. He, Z. Zhang, Y. Shi, K. Shirahige, P. Pasero, X. Shen, Involvement of a chromatin remodeling complex in damage tolerance during DNA replication, Nat. Struct. Mol. Biol. 16 (2009) 1167-1172. 
[17] M.S. Huen, J. Chen, The DNA damage response pathways: at the crossroad of protein modifications, Cell Res. 18 (2008) 8-16.

[18] A.J. Morrison, J.A. Kim, M.D. Person, J. Highland, J. Xiao, T.S. Wehr, S. Hensley, Y. Bao, J. Shen, S.R. Collins, J.S. Weissman, J. Delrow, N.J. Krogan, J.E. Haber, X. Shen, Mec1/Tel1 phosphorylation of the INO80 chromatin remodeling complex influences DNA damage checkpoint responses, Cell 130 (2007) 499-511.

[19] R.C. Conaway, J.W. Conaway, The INO80 chromatin remodeling complex in transcription, replication and repair, Trends Biochem. Sci. 34 (2009) 71-77.

[20] L.Y. Tang, N. Deng, L.S. Wang, J. Dai, Z.L. Wang, X.S. Jiang, S.J. Li, L. Li, Q.H. Sheng, D.Q. Wu, R. Zeng, Quantitative phosphoproteome profiling of Wnt3a-mediated signaling network: indicating the involvement of ribonucleoside-diphosphate reductase M2 subunit phosphorylation at residue serine 20 in canonical Wnt signal transduction, Mol. Cell. proteomics 6 (2007) 1952-1967.

[21] N. Dephoure, C. Zhou, J. Villen, S.A. Beausoleil, C.E. Bakalarski, S.J. Elledge, S.P. Gygi, A quantitative atlas of mitotic phosphorylation, Proc. Natl. Acad. Sci. U.S.A. 105 (2008) 10762-10767.

[22] T.A. Day, K. Palle, L.R. Barkley, N. Kakusho, Y. Zou, S. Tateishi, A. Verreault, H. Masai, C. Vaziri, Phosphorylated Rad18 directs DNA polymerase eta to sites of stalled replication, J. Cell Biol. 191 (2010) 953-966.

[23] E.J. Park, S.K. Hur, and J. Kwon, Human INO80 chromatin-remodelling complex contributes to DNA double-strand break repair via the expression of Rad54B and XRCC3 genes, Biochem. J. 431 (2010) 179-187.

[24] S.K. Hur, E.J. Park, J.E. Han, Y.A. Kim, J.D. Kim, D. Kang, J. Kwon, Roles of human INO80 chromatin remodeling enzyme in DNA replication and chromosome segregation suppress genome instability, Cell. Mol. Life Sci. 67 (2010) 2283-2296.

[25] Y. Jiang, X. Wang, S. Bao, R. Guo, D.G. Johnson, X. Shen, L. Li, INO80 chromatin remodeling complex promotes the removal of UV lesions by the nucleotide excision repair pathway, Proc. Natl. Acad. Sci. U.S.A. 107 (2010) 17274-17279.

[26] C. Muchardt, J.C. Reyes, B. Bourachot, E. Leguoy, M. Yaniv, The hbrm and BRG-1 proteins, components of the human SNF/SWI complex, are phosphorylated and excluded from the condensed chromosomes during mitosis, EMBO J. 15 
(1996) 3394-3402.

[27] S. Sif, P.T. Stukenberg, M.W. Kirschner, R.E. Kingston, Mitotic inactivation of a human SWI/SNF chromatin remodeling complex, Genes Dev. 12 (1998) 2842-2851.

[28] J.C. Randell, A. Fan, C. Chan, L.I. Francis, R.C. Heller, K. Galani, S.P. Bell, Mec1 is one of multiple kinases that prime the Mcm2-7 helicase for phosphorylation by Cdc7, Mol. Cell 40 (2010) 353-363.

[29] C. Masutani, R. Kusumoto, A. Yamada, N. Dohmae, M. Yokoi, M. Yuasa, M. Araki, S. Iwai, K. Takio, F. Hanaoka, The XPV (xeroderma pigmentosum variant) gene encodes human DNA polymerase eta, Nature 399 (1999) 700-704.

[30] R.K. Chiu, J. Brun, C. Ramaekers, J. Theys, L. Weng, P. Lambin, D.A. Gray, B.G. Wouters, Lysine 63-polyubiquitination guards against translesion synthesis-induced mutations, PLoS Genet. 2 (2006) e116.

\section{Figure legends}

\section{Figure 1 hINO80 is involved in PCNA ubiquitination.}

HeLa cells were transfected with either non-targeting control (N.C.) siRNA or hINO80 targeting siRNA and cultured for 72 or 96 h. (A) Whole-cell lysates of these cells were analyzed for hINO80 expression using Western blotting. PCNA expression was also analyzed as a loading control. (B) Whole-cell lysates of these cells were analyzed for PCNA ubiquitination using Western blots. The long exposure of Western blotting was conducted to visualize PCNA ubiquitination. (C) HeLa cells were transfected with control or hINO80 targeting siRNA. Forty-eight hours after the first transfection, these cells were transfected with empty vector or with the hINO80 expression construct and cultured for $48 \mathrm{~h}$. PCNA ubiquitination was analyzed on Western blots of whole-cell lysates of these cells.

\section{Figure 2 hINO80 is necessary for recruitment of Rad18 to site of DNA damage.}

HeLa cells were transfected with control or hINO80 targeting siRNA and cultured for $48 \mathrm{~h}$. The cells were treated with 30 $\mathrm{J} / \mathrm{m}^{2} \mathrm{UV}$, and then cultured for $2 \mathrm{~h}$. (A) Rad18 expression was analyzed on Western blots of whole-cell lysates. (B) These 
cells were fixed with $\mathrm{MeOH}$ for 15 min. After permeabilization and a blocking procedure, the fixed cells were stained with anti-rad18 antibody (green) (C) The nuclear dots intensity of Rad18 was quantified with IMAGE J software. In each type of cell, 50 cells were randomly chosen, and the mean value of Rad18 immunofluorescence in the nuclei was quantified. Box plot showed the quantification of the nuclear dots intensity of Rad18. The dot intensity was defined as maximum value of intensity. $*$ P value $<10^{-4}$

\section{Figure 3 C-terminal region of hINO80 is phosphorylation.}

(A) Whole-cell lysates of HEK-293T cells were treated with $\lambda$-phosphatase, and analyzed for hINO80 expression by Western blotting. (B) Schematic diagrams of human INO80 full-length protein and the constructed fragments used in this study. (C) HEK-293T cells were transfected with one of three constructs encoding a hINO80 deletion mutant and cultured for $48 \mathrm{~h}$. Whole-cell lysates of these cells were analyzed for expression of the each hINO80 fragment by Western blotting. (D) HEK-293T cells were transfected with an expression construct encoding the C-terminal region of hINO80 and cultured for $48 \mathrm{~h}$. Whole-cell lysates of these cells were treated with $\lambda$ phosphatase, and analyzed for expression of the hINO80 C-terminal fragment by Western blotting. (E) Schematic diagrams of phosphorylation-defective mutants of the hINO80 C-terminus used in this study. (F) HEK-293T cells were transfected with one of these mutant constructs and cultured for 48 h. Whole-cell lysates of these cells were analyzed for expression of the each hINO80 fragment by Western blotting.

\section{Figure 4 Phosphorylation of hINO80 is involved in DNA damage tolerance.}

HEK-293T cells were transfected with pcDNA3.1 hINO80 or the PM-hINO80 expression construct and cultured for $48 \mathrm{~h}$.

The cells were exposed to $300 \mu \mathrm{M}$ hydroxyurea (HU) for $14 \mathrm{~h}$, released into drug-free medium for $8 \mathrm{~h}$, exposed again to $\mathrm{HU}$ for $14 \mathrm{~h}$ again, and then released again. (A) PCNA ubiquitination was analyzed on Western blots of whole-cell lysates from these cells. (B) Expression of $\gamma \mathrm{H} 2 \mathrm{AX}$ was analyzed on Western blots of whole-cell lysates from these cells; PCNA expression was also analyzed as a loading control on these blots. 
Figure's

Figure 1

A

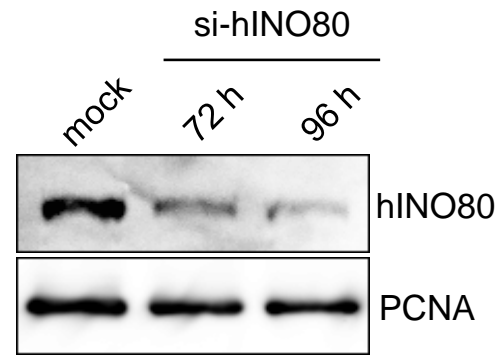

B

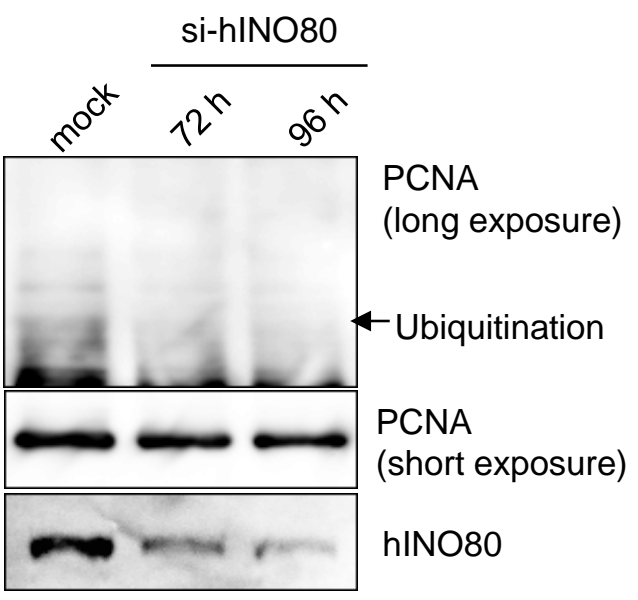

C

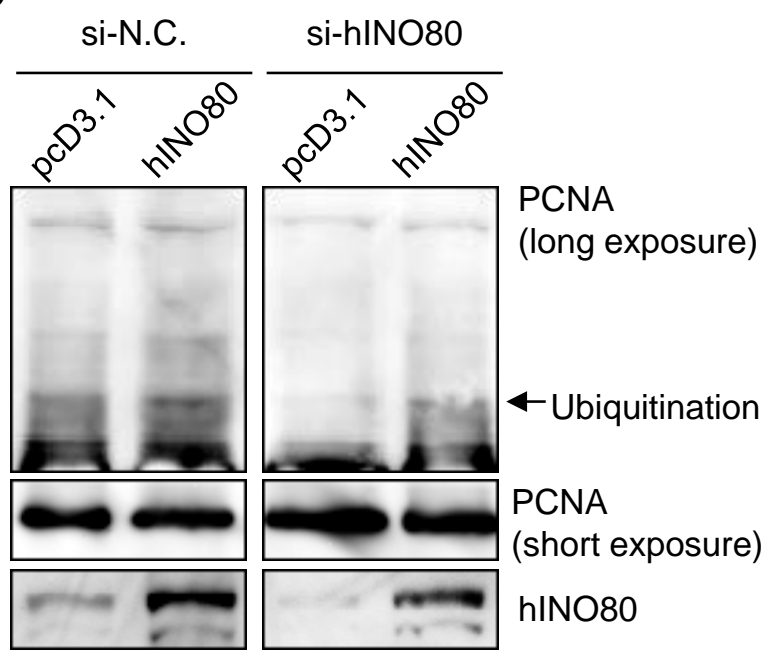


Figure 2

A

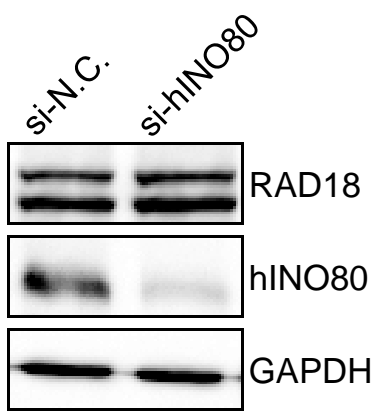

B

si-N.C.

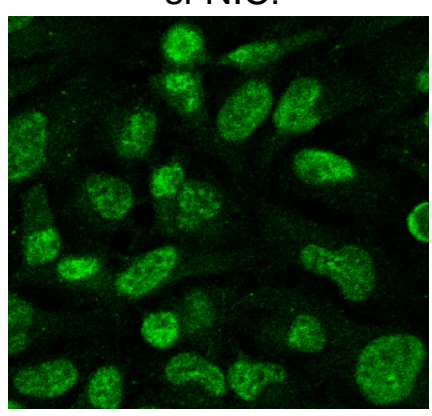

si-hINO80

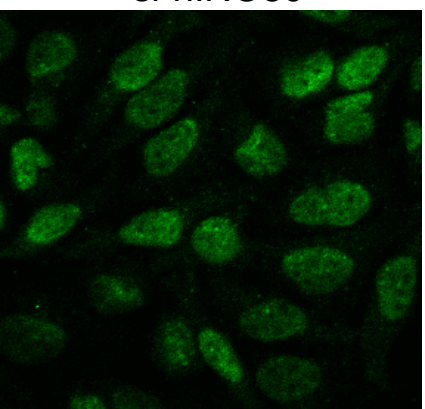

C

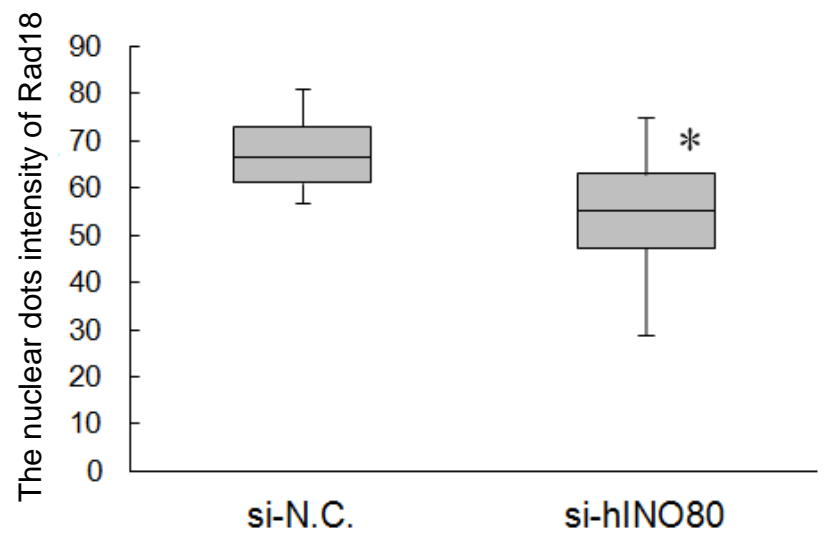


Figure 3

A

$\lambda$ PPase

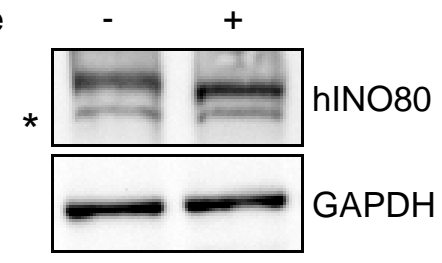

B hINO80 DEAQ

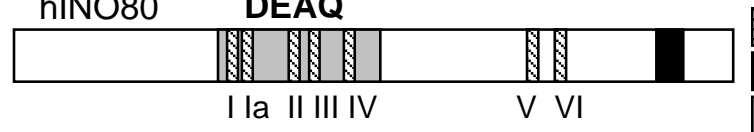

$$
\text { C ter } \Delta
$$

Helicase motif Bromo domain

SNF2 N-terminal domain

1-1218

.
C

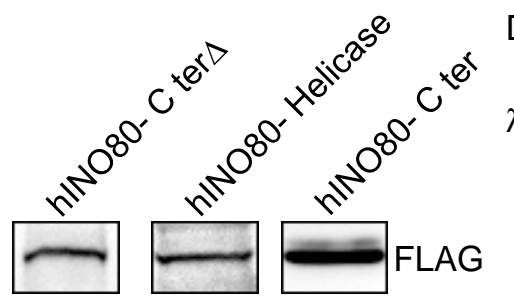

D

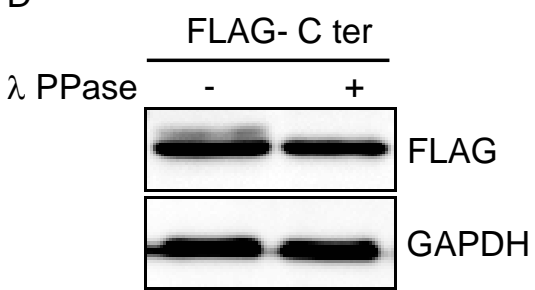

E

S1490 S1516

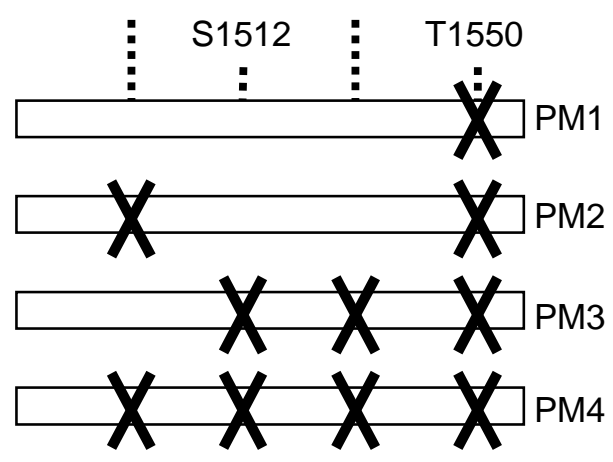

F

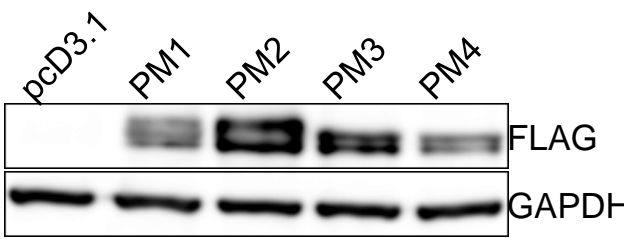

540-1218

C ter

1219-1556

Helicase
Cter

1219-1556

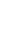


Figure 4

A

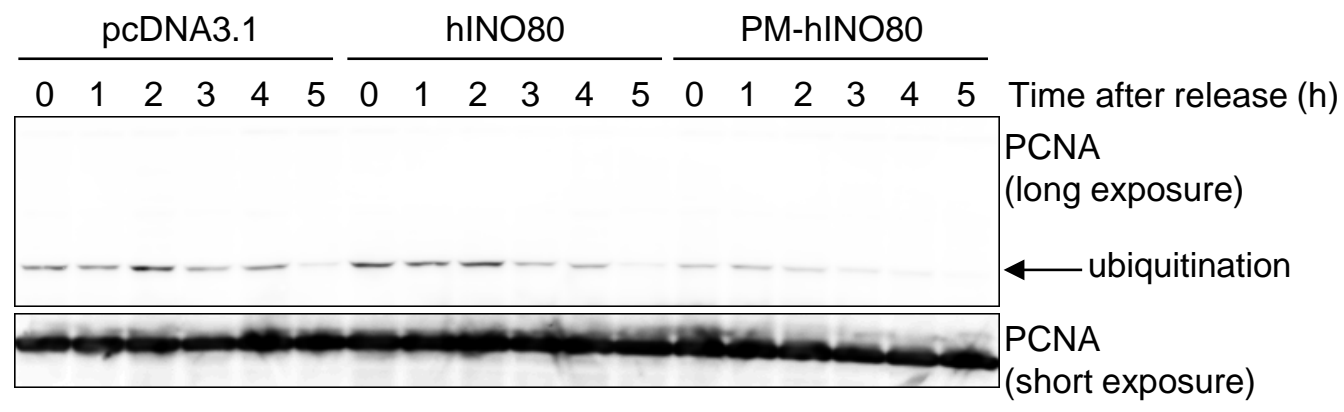

B

\begin{tabular}{|c|c|c|c|c|c|c|c|c|c|c|c|c|c|c|c|c|c|}
\hline \multicolumn{6}{|c|}{ pcDNA3.1 } & \multicolumn{6}{|c|}{ hINO80 } & \multicolumn{5}{|c|}{ PM-hINO80 } & \multirow{3}{*}{$\begin{array}{l}\text { Time after release }(h) \\
\gamma \mathrm{H} 2 \mathrm{AX}\end{array}$} \\
\hline 0 & 1 & 2 & 3 & 4 & 5 & 0 & 1 & 2 & 3 & 4 & 5 & 0 & 12 & 3 & 4 & 5 & \\
\hline - & - & - & - & $=$ & - & & & & & & & & & & & & \\
\hline
\end{tabular}

